was obviously undesirable to interfere with the process of nature beyond assisting in the removal of the necrotic tissue, for any incision made with a view of relieving the constriction would necessarily open up the barrier existing between the healthy and infected regions, thereby exposing the patient to the risk of general peritonitis, whereas, if the necrotic tissue was allowed to separate or was scraped away, every care being taken to disinfect and purify the locality, one might reasonably anticipate a favourable issue, even if it involved the fixation of the omental stump to the abdominal parietes. If after the wound has healed the patient should complain of pain and threatening obstruction from restricted peristalsis, an operation for the relief of the deep adhesions can be resorted to under more favourable auspices.

CASE 2.-A woman aged sixty-two years was admitted to the Wigram Ward on Jan. 2nd, 1896, suffering from a large irreducible femoral hernia on the right side extending upwards and outwards over Poupart's ligament. The origin of this protrusion dated back to a confinement thirty-eight years ago. She has occasionally worn a truss, but at variable intervals has had severe attacks of pain localised to the hernial sac and evidently due to a limited peritonitis. The tumour has increased in size considerably during the last seven or eight months. On admission it was hard and irreducible, but not tender to the touch. She has also suffered much from general dyspepsia and attacks of vomiting. On operation I found adherent to the sac a large mass of omental tissue which had undergone a sclerotic change as the result of the repeated attacks of inflammation; it was completely shut off from the general peritoneal cavity. Running through the centre was a tubular body, which proved on dissection to be a long vermiform appendix. This and the dense mass of omentum were removed on a level with the femoral opening and the wound healed in a few days.

In comparing this case with the other I would again remind you of what I stated at the beginning of the lectureviz., that nature had here effected a cure before I interfered, and that the operation merely consisted in removing a superfluous mass of changed omentum and the appendix, which had long since been isolated from the abdominal cavity. Indeed, so firm was the plugging of the femoral canal that it was not necessary to disturb it in any way. At the same time it must be evident to you all that this woman was for a long time in very considerable danger with a prolapsed vermiform appendix in addition to the omental protrusion. At any moment a coil of gut might have passed into the sac and led to strangulation, or a similar condition of acute obstruction might have been induced by kinking of the gut from dragging or twisting of the omental connexions. Again, the prolapsed appendix might have become acutely inflamed, although this condition is extremely rare.

The presence of the vermiform appendix in a hernial sac is not a common occurrence, although not a few cases have been recorded. Personally, although I have operated on hundreds of cases of hernia I have not met with this condition more than half a dozen times. Some years ago I was called in by Mr. Alfred J. Bell of St. John's Wood to operate on an old lady over eighty years of age who was suffering from acute strangulated femoral hernia, and I there found the appendix in the sac in addition to a coil of intestine. The removal of the former and the usual operation of radical cure were followed by an uneventful recovery. No doubt in this latter case the appendix had been the first tenant of the hernial sac and had paved the way for a prolapse of the bowel, which had subsequently become strangulated. In the former case no coil of bowel happened to become involved.

In conclusion, I must repeat that these two cases are of a veny exceptional character and must not be looked upon as in any way typical of the ordinary run of cases of incarcerated or strangulated hernia with which you will have to deal in your practice.

Wigan Medical Soctetr.-The ordinary meeting of this society was held on June 4th, Mr. C. R. Graham, President, being in the chair.--Dr. Judson S. Bary, assistant physician to the Manchester Royal Infrimary, read a paper on the Diagnosis of Functional from Organic Disease of the Nervous System.-A discussion followed, in which Mr. Hugh E. Jones, Mr. Cowan, Dr. I. P. White, Mr. Benson, and the President took part.A cordial rote of thanks was passed to Dr. Bury for his able paper.-Dr. Bury replied!

\section{A CASE OF CONFLUENT SMALL-POX IN A LASCAR.}

BY ALEXANDER MACPHAIL, M.B., C.M.GLASG., DHMONSTRATOR OF ANATOMY, GLASGOW UNIVERSITY.

2. THE following notes, chart, and drawings form the record of a case of confluent small-pox which ran its course to a fatal issue on the high seas between the ports of Colombo and Suez, and are mainly interesting from the fact that a complete record of such a case is not often obtainable, the patient having been one of a Lascar crew. A secondary interest attaches to the record in that I was at the time surgeon on board the ss. Clan Mackenzie, and had not previously seen a case of small-pox, so that the diagnosis had to be made and the case conducted without such aid as anyone familiar with the disease might derive from early signs ; and it was only determined after a careful study of the notes taken from day to day, which are given below, together with some remarks on the chief points of interest.

On Sept. 18th, 1894, a man aged thirty-five years first complained of headache and pains in the back and limbs. The skin was hot and dry. The temperature was scarcely febrile in the morning, but reached $101^{\circ} \mathrm{F}$. by the evening. He was given quinine in ten-grain doses. On the 19th the temperature was steadily rising; there was profuse sweating. The pains were general and more severe. The patient was also suffering from depression and sleeplessness. On the 20th the fever was more marked and the pain less. There was severe vomiting shortly after a dose of quinine in the morning, and phenacetin was substituted. The vomiting reappeared several times in the course of a few hours. On the 21st, although there were symptoms of greater ease, the morning temperature rose still higher, with a fall of $2^{\circ}$ after exhibition of phenacetin again at midday, when the patient drew attention to a few spots on his forehead, like ordinary pimples, but hard, while a few similar spots were felt on the right cheek. These were not regarded as of much importance, and no rash conld be seen; but on the evidence of continued pyrexia and inexplicable fits of vomiting the patient was isolated, disinfectant measures were adopted, and the temperature was taken more frequently. On the 22nd distinct papules appeared all over the body, with very slight signs of congestion, but in almost all cases giving a "shotty" feeling to the touch. The temperature reached a crisis at $104.4^{\circ}$ in the afternoon and fell again towards evening. Isolation and disinfectant measures were more strictly enforced on the first suspicion of small-pox. On the 23rd there was a decided lysis of the temperature almost to normal, with slight exacerbation in the afternoon. The symptoms were much relieved, and the pulse, though not notably accelerated previously, became almost normal in number and fulness of beat. The eruption was still nondescript, some of the spots showing an advance towards breaking and scabbing and others to surface blackening. On the 24th the temperature was still oscillating at the lowest febrile degree. The individual spots were more prominent, broader, and flat, and dull chocolate in colour. (See Fig. 1.) Sleeplessness was also present for which bromide of potassium was exhibited. On the 25th the morning temperature was normal, but febrile again at night. Delirium was very marked during the night, the eruption assuming the above-mentioned characters all over. Acupuncture of the flat elevations revealed serous fluid contents. There was slight confluence on the forehead and cheeks, the lips and tongue were swollen and marked by distinct papules, and one or two clear vesicles were seen on the sides of the tongue. On the 26th the temperature was oscillating. There were a few distinct pustules on the face and in the fold of the groin. The whole face was much swollen and the pulse became rapid and thin. On the 27th the temperature was increasingly febrile and the pulse was 112, weak, and dicrotic. Noisy and incessant delirium was present, the spots all over the body becoming pustular (Fig. 2) The eyelids were matted with purulent matter and there was a discharge from the nose. The patient showed great thirst. The urine was very concentrated. Zinc powder and perchloride of mercury lotion were applied externally, while digitalis and stimulant were given internally. On the 28th all the symptoms were less severe in the morning and throughout the day; the pulse, although weak, was less 
rapid; the temperature was $99 \cdot 8^{\circ}$ in the morning, rising again during the afternoon; the delirium was gone. Death took place unexpectedly at.night, the patient having spoken cheerily to his comrades through the partitions a few minutes previously.

Remarks. - The duration of the period of incubation can be very definitely fixed, as the patient had been, on his own information, in direct contact with a case of small-pox on Sept. 5th and 6th, his own initial symptoms appearing on the morning of Sept. 18th. During this time he had never complained of any indisposition, although the captain had remarked on a drowsiness and inactivity quite unusual to the patient. The first complaint was made in company with three other native servants who had got a wetting the previous night and had thereafter gone to sleep for a few hours on the saloon floor in order to be at hand when the ship arrived at Colombo. They all complained of symptoms which at once suggested the early stages of an attack of ague-viz., headache, pains in the back and lower limbs, and hot, dry skin, the temperature, though above normal, in no case being febrile as yet. Malarial fever, or simply

FIG. 1.

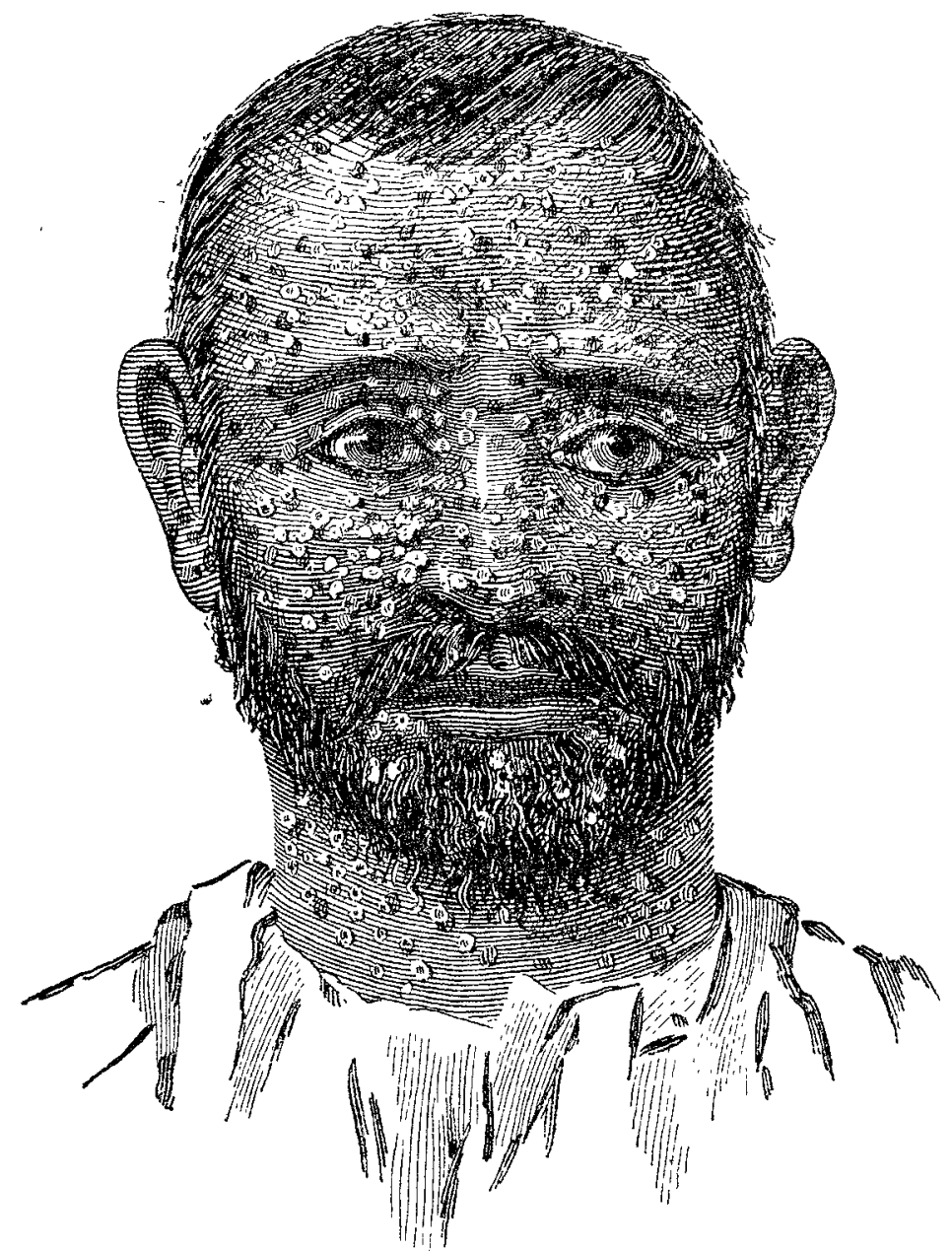

Fifth day of eruption: vesicular stage.

"fever," as it is called by them, is always smouldering in a Lascar crew, and as similar causes had accounted for several well-marked cases in the previous voyage, the diagnosis of malarial fever was considered secure and the routine treatment-by quinine sulphate in ten-grain doses every few hours-was adopted. The relief following the exhibition of quinine in all the cases, with this single exception, seemed to justify this diagnosis, and it was fully anticipated that this case too would soon subside under its influence. On the contrary, the temperature curve was still rising (although unattended by any new symptom) on the second day, and continued so till the morning of the third day, when severe vomiting and retching set in. This was at first attributed to the usual dose of quinine, but in spite of phenacetin having been substituted it came on several times during the day and was seemingly independent of any stomach irritation, there being only a slight emesis of bile-coloured fluid, pointing rather to a constitutional cause, associated no doubt with the invasion of the disease. The temperature chart gradually evolved a curve resembling that typical of small-pox, a somewhat abrupt rise from normal to $104 \cdot 4^{\circ}$, which crisis was reached on the fifth day of illness and one day after the first appearance of the exanthem. This was interrupted on two occasions by phenacetin, which produced a transitory fall. Coincident with the outbreak of the papules all orer the body a distinct lysis set in, the temperature becoming almost subfebrile in the course of twenty-four hours, and, oscillating there for two days, on the morning of the third day reached normal. The secondary curve, which began to derelop on the same day, although intermittent, showed a steady rise, reflecting the process of pustulation and consequent blood-poisoning. The pulse in the earlier stages presented no feature out of proportion to the degree of ferer present, and only when the secondary fever set in was there any notable feature; then its rate increased as its strength diminished, its character becoming distinctly thready. These qualities were markedly changed by the administration of trro doses of fifteen minims each of tincture of digitalis and some stimulant. From an early date the exanthem was regarded as significant of a specific fever, but, in the absence of previons experience of small-pox, was not for some time confidently held to indicate the presence of this disease. In the early stage the spots were distinctly "shotty," but might uave passed

FIG. 2.

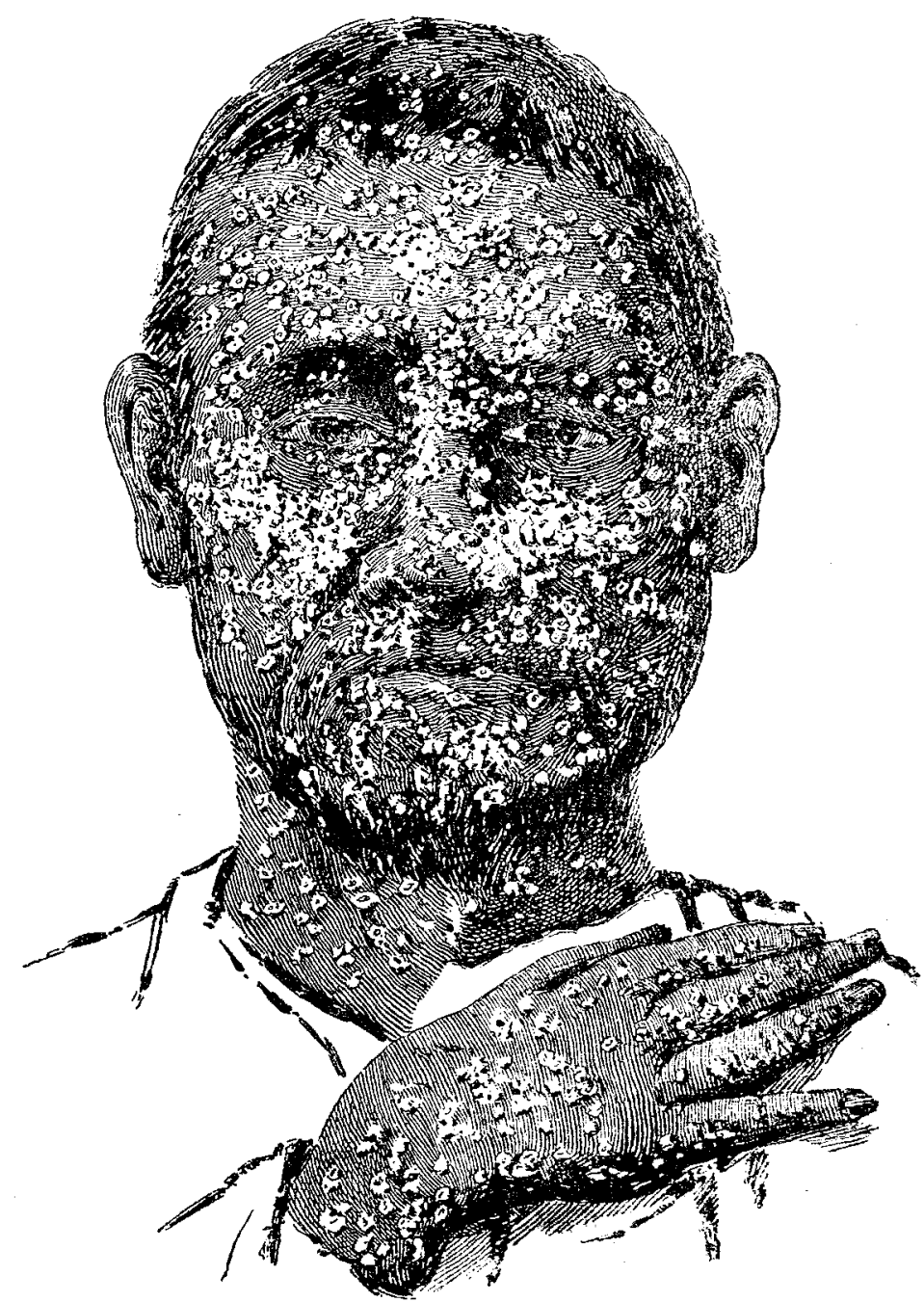

Seventh day of eruption: pustular stage.

except for the absence of a distinct "core" as ordinary pimples. The rapidly increasing area over which they began to appear and their transition into llat, hard elevations, mostly without any manifest congestion, led to a crossexamination of the patient as to his erer having seen a similar eruption. He said he harl, and was sure that it was a certain disease common among natires of Inclia, to which he gave the name " hoom," but which I lare been unable to identify farther than that it is sometimes used to describe chicken-pox and is more loosely applied to many skin affections. When asked if he was familiar with small-pox the important fact was elicited that he had been taying for a few days, when the ship was lying at Calcutta, with an uncle stricken with small-pox there, and, in entire ignorance of any theory of infection, had been in constant communication with him as with any ordinary inralid. Thongh important this was not regarded as cunclusire nroof, for until it had assumed the characters shown in Fig. 1 the eruption did not seem to conform to the usual description of a smallpox vesicle; indeed, it did not suggest the presence of fluid 
at all; but presented a slightly elevated, usually oblong after every visit. Disinfection in the cabin set apart for surface, quite firm to the touch and of a dull chocolate the patient was effected by means of a moist sheet colour. Other characters common to the small-pox sprinkled with carbolic solution bung over the doorway. exanthem were, however, present-viz., distinct depres- All food, \&c., was passed to me through the barricades and sion as if from drawing together of the centre in some the dishes washed with carbolic solution after use. After cases, in others a softening of the surface with scabbing, the death of the patient his cabin was washed down with the without any evidence of the usual cycle of vesiculation steam hose and afterwards scrubbed with carbolic acid, and pustulation. Distinct vesiculation such as is suggested, while both it and the forecastle adjoining were well fumito quote any one of the recognised authorities, by gated. It was with difficulty that the Lascars could be the description "hemispherical in form and opaline in argued into leaving to me the preparation of the body for appearance" was not manifestly present, and, as many burial, and, while conceding this, they insisted on holding a burial service on deck. Every precaution

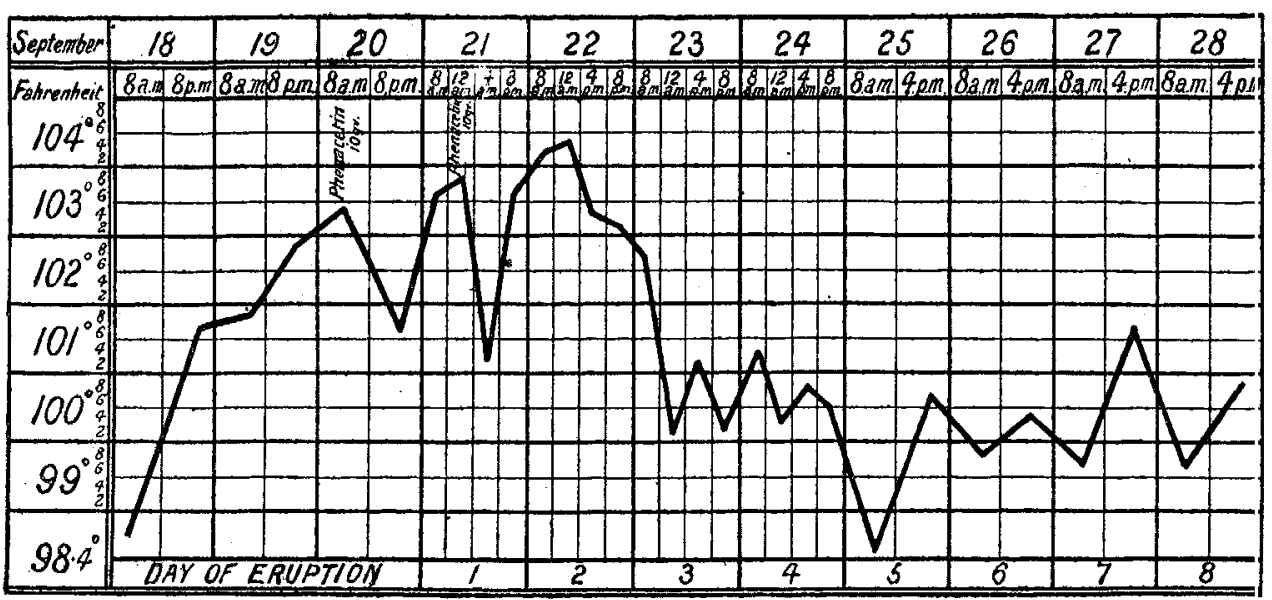

Chart showing rise until Sept. 22nd, when the temperature was over $104^{\circ}$ and there were spots all over the body. Coincidently with the appearance of the rash the temperature fell.

other indications pointed to the eruption being that of smallpox, proof of vesiculation was sought for by puncture of one of these flat elevations; the result was the escape of a small amount of serous fluid removing all doubt of the condition. Previous experience might have lel to the same conclusion at an earlier stage, but as the decision of the true nature of the case was one of immense importance to the ship's company it was delayed by the outlook for such a condition as the common description of resiculation had suggested. Subsequently confluence of these areas and the appearance of pustulation took their usual course, producing the appearance seen in Fig. 2, the only additional fact worthy of remark being the presence of black scabs among the pustules indicating unusually speedy maturation here and there. The prognosis was not at first grave, the only disturbing factors in the early stages being the great tropical heat both by day and night and the early appearance of delirium. The extensive confluence in the later stages indicated a serious outlook. Curiously, what appeared to be the turning-point towards recovery must have been a final struggle on the part of nature, for on the day preceding his death the patient had for the first time expressed himself as "getting better," and his general condition had, moreover, apparently im. proved.

With regard to treatment, little could be done medicinally. Quinine evidently had little or no effect in controlling the initial fever, although phenacetin in equal doses (ten grains) took effect upon two occasions. Bathing the eyes with a weak solution of perchloride of mercury gave much relief, as also did the application to the face of boric and zinc powder on a mask of boracic lint. Milk diet was adopted on the first evidence of continued fever; condensed milk only being available, this was given with fine-boiled rice three times daily. Alcoholic stimulant, for which there seemed no indication till the onset of the secondary fever, was at first refused on religious grounds (its use involving loss of caste), but was insisted on later when strength was evidently failing with good, although temporary, result. Isolation was at first no easy matter owing to the Lascars, having no idea of contagion, trying to communicate with the patient; the cabin had to be barricaded so that entrance could only be obtained in sight of the watch on the bridge the delirium of the patient added another danger, as on one occasion he rushed out on deck and was got back with difficulty. On my part, too, there was great need for caution, as the case had to be taken full charge of in addition to my ordinary duties. To allow of this special clothing was set apart for my visits; my cabin was occasionally fumigated, and a hot bath, impregnated with Condy's fluid, was taken

1 Hilton Fagge: Text-book of Principles and Practice of Medicine, third edition, p. 202 . was taken by the captain, who stopped

the ship and ran her broadside to the wind. Three of the Lascars who bore small-pox marks were chosen to remore the body from the cabin (where it had previously been wrapped in well. carbolised sheets and canvas) to the leck, where they deposited it on one side; the whole of the Lascar crew assembled on the other at a distance of some four yards while the service, lasting a quarter of an hour, was conducted. After the body was consigned to the deep additional precautions were taken to disinfect those who had acted as bearers.

These facts are given in some detail because they seemed to bear a sequel for thirteen days afterwards (the ship having escaped quarantine at Suez, Port Said, and Malta) a young Lascar fireman was struck down by symptoms which soon left no doubt that he was suffering from small pox. The coincidence of this lapse of time-corresponding so closely to the usual period of incubation-with the onset of his symptoms seemed to point to the burial service on deck as a loophole in the disinfectant precautions. This was all the more disappointing as the danger of its being so had been clearly recognised, and though the service seemed inevitable great care had been taken to isolate the participants in the service from the deceased as far as possible. Fortunately for the sequel, this second case was of a much milder type and made a good recovery on reaching the Thames.

Glasgow.

\section{FURTHER NOTE ON THE TREATMENT OF} TAPEWORM.

Bx LESLIE OGILVIE, B.Sc., M.B. EDIN., M.R.C.P.LoND. PHYSICIAN TO THE PADDINGTON-GREEN CHILDREN'S HOSPITAL.

IN The LAxCET of Aug. 4th, 1894, I contributed a note on the Treatment of Tapeworm. As six cases-two children and four adults-which I have since seen had been previously treated unsuccessfully I venture to think that my further experience may be of interest. The line of treatment indicated in my previous communication was successfully followed in all of these cases, but I desire to refer to two of them as they appear to me to corroborate the views I then expressed as to the necessity of preparing the patient before administering the vermifuge and the absence of danger in giving the considerable doses of the liquid extract of malefern which I recommended if it is speedily washed from the bowel.

CASE 1.-The patient was a little girl aged seven years who had undergone treatment many times during three years, but without success. She had been given repeated small doses of about fifteen minims of the liquid extract of male-fern, and although numerous segments of the worm were passed after each course of treatment they re-appeared in the stools in two or three months, when the child herself would again ask for "fern," as she felt sick and irritable and had abdominal discomfort. The child dreaded the administration of the drug as it always made her so ill for some days, but she preferred this to the unpleasant sensations produced by the presence of the parasite. The medical man in attendance was afraid to give the large doses recommended by me-one drachm at $8 \mathrm{~A} . \mathrm{M}$. and another drachm or half a drachm at 9 A.M. - as he argued that if fifteen 